\title{
CHANGES OF ELECTRONIC STRUCTURE OF SnTe DUE TO HIGH CONCENTRATION OF Sn VACANCIES
}

\author{
J. MašEK and D.N. NuzhNyJ
}

Institute of Physics, Academy of Sciences of the Czech Republic Na Slovance 2, 18040 Praha 8, Czech Republic

Non-stoichiometric $\mathrm{Sn}_{1-y} \mathrm{Te}$ is a strongly degenerated $n$-type semiconductor. This is important for understanding unusual features of magnetic behaviour of $\mathrm{Sn}_{1-x} \mathrm{Gd}_{x} \mathrm{Te}$ where the relative positions of the Fermi energy and the atomic $d$-level of Gd govern the exchange coupling. The influence of the $\mathrm{Sn}$ vacancies on the band structure cannot be neglected if their concentration reaches a few atomic percent. We address this problem by using a tight-binding coherent potential approach and show that although the character of the bands remains unchanged, they are modified so that $\varepsilon_{d}$ can come out above the heavy-hole band.

PACS numbers: $71.20 . \mathrm{Nr}, 71.55 . \mathrm{Jv}$

\section{Introduction}

Unusual features of the exchange coupling between local magnetic moments at $\mathrm{Gd}^{3+}$ ions in $\mathrm{Sn}_{1-x} \mathrm{Gd}_{x} \mathrm{Te}$, and the recent discussion about a new mechanism of $f-f$ exchange interactions in this semimagnetic semiconductor [1] attracted a new attention also to the host material, i.e. SnTe.

SnTe is a narrow-gap semiconductor with a band structure typical of IV-VI compounds. It is characterized by five occupied valence bands and a direct gap at the $L$-point. Other maxima of the valence band at $\Sigma$ and $\Delta$ lie just below the top of the band. In contrast to the tetrahedral semiconductors, the hybridization is less important here and the bands have their well-defined orbital origin (anion and cation $s$ - and $p$-states). The only exception are the light-hole states close to the valence band top where the hybridization of anion $p$-states and cation $s$-states is important and makes the position of the band edge sensitive to a substitution in the cation sublattice [2].

The $\mathrm{SnTe}$ crystals are known to contain a very high number of $\mathrm{Sn}$ vacancies which are assumed to be acceptors with a zero activation energy. The concentration $y$ of the vacancies may be as high as 6 atomic percent. At this level of "doping" the hole density $p$ reaches its maximum value of about $2 \times 10^{21} \mathrm{~cm}^{-3}$ and the Fermi energy is located at around $0.2 \mathrm{eV}$ below the band edge, i.e. below the edge of the heavy hole band at $\Sigma$. 
Story et al. [1] noticed that the distance of the Fermi energy from the valence-band top defines a reasonable energy scale within which the arrangement of relevant energy levels can decide about the physical behaviour of the material. For $\mathrm{Sn}_{1-x} \mathrm{Gd}_{x} \mathrm{Te}$, it is assumed that the exchange coupling between local moments at $\mathrm{Gd}^{3+}$ ions (formed by $f$-states) is mediated by the unoccupied $d$-states which can hybridize with the band states. This mechanism is efficient only if the $d$-level $\varepsilon_{d}$ falls into the heavy-hole band and the Fermi energy approaches it from below. This seems to happen for $x<0.05, p \approx 2 \times 10^{20}$ when the enhanced antiferromagnetic (AF) exchange interaction is observed. If $\varepsilon_{d}$ moves into the light-hole band (for $x>0.05$, due to the band structure reconstruction with increasing $\mathrm{Gd}$ content), or if it is not resonant with $E_{\mathrm{F}}$ (for $x<0.05$, but $p \gg 2 \times 10^{20} \mathrm{~cm}^{-3}$, due to high "doping"), the proposed mechanism of the mediated exchange is ineffective and the $\mathrm{AF}$ coupling remains weak.

The theory, however, implies two non-trivial assumptions that:

(i) the line width of the $d$-state due to the hybridization is much smaller than the energies in play, i.e. than $c a .100 \mathrm{meV}$;

(ii) the vacancies act only as the acceptors and do not influence the band structure. While the former assumption can be simply checked, using e.g. the Harrison prescription for the $s p-d$ hybridization [3], the latter needs a more careful treatment. This is the aim of the present work.

We assume that the vacancies are randomly distributed in the cation sublattice. We describe them as atoms $\square$ with very high energy barriers, and represent the non-stoichiometric compound $\mathrm{Sn}_{1-y} \mathrm{Te}$ as a mixed crystal $\mathrm{Sn}_{1-y} \square_{y} \mathrm{Te}$. The density of states is calculated by using the coherent potential approach (CPA). Starting from the CPA, we also derive a mean-field approximation providing an effective band structure of the non-stoichiometric crystal.

\section{Tight-binding version of the coherent potential approximation}

To describe the valence and the conduction bands of $\mathrm{SnTe}$, we use a tight-binding model with a minimum basis consisting of $s$ - and $p$-states. We identify the diagonal matrix elements with the atomic energy levels at both anions and cations $\left(\varepsilon_{s}^{\mathbf{A}}, \varepsilon_{p}^{\mathbf{A}}, \varepsilon_{s}^{\mathbf{C}}, \varepsilon_{p}^{\mathbf{C}}\right)$. According to Ref. [4], we consider the interatomic coupling not only between the nearest neighbours, but also for adjacent anion-anion (cation-cation) pairs.

In the non-stoichiometric crystals, we assume that the hopping elements remain unchanged, and that the local barriers at the vacancies are represented by high enough $\varepsilon_{s}^{\square}$ and $\varepsilon_{p}^{\square}$. The randomness in the mixed crystal $\mathrm{Sn}_{1-y} \square_{y} \mathrm{Te}$ is restricted only to the cation sublattice so that the CPA procedure applies only to the cations. The self-energy, replacing random atomic levels in the configurationally averaged Green function

$$
G(z) \stackrel{\text { def }}{=}\left\langle(z-H)^{-1}\right\rangle_{\mathrm{av}}=\left[z-H_{0}-\Sigma(z)\right]^{-1},
$$

has two independent matrix elements, $\Sigma_{s}^{\mathrm{C}}(z)$ and $\Sigma_{p}^{\mathrm{C}}(z)$. The corresponding local Green functions are the matrix elements of $G(z)$ at (any) cation site $i$,

$$
G_{s}^{\mathrm{C}}(z)=\langle i, s|G(z)| i, s\rangle \equiv \frac{1}{z-\Sigma_{s}^{\mathrm{C}}(z)-\Gamma_{s}(z)},
$$




$$
G_{p}^{\mathrm{C}}(z)=\langle i, p|G(z)| i, p\rangle \equiv \frac{1}{z-\Sigma_{p}^{\mathrm{C}}(z)-\Gamma_{p}(z)} .
$$

The CPA condition has a simple form expressing the local matrix elements of the averaged Green function as the weighted sum of the local Green functions for cation atoms $(C)$ and vacancies $(\square)$ immersed into the effective medium

$$
\frac{1}{z-\Sigma_{s, p}^{C}(z)-\Gamma_{s, p}(z)}=\frac{1-y}{z-\varepsilon_{s, p}^{\mathrm{C}}-\Gamma_{s, p}(z)}+\frac{y}{z-\varepsilon_{s, p}^{\square}-\Gamma_{s, p}(z)} \text {. }
$$

\section{Mean-field model for the band structure of $\mathbf{S n}_{1-y} \square_{y} \mathbf{T e}$}

Besides the full CPA treatment, based on a self-consistent solution of Eqs. (1)-(4), we shall use also a simplified scheme for the construction of the self-energy. Let us consider

(i) vacancy sites inaccessible for electrons, i.e. $\varepsilon_{s, p}^{\square} \rightarrow \infty$;

(ii) the atomic limit $\Gamma_{s, p}(z) \approx 0$, i.e. the ionic nature of the bonding with only weak mixing of the cation and anion states.

Under these assumptions, Eq. (4) results in an explicit expression of the self-energy

$$
\Sigma_{s, p}(z)=-\frac{y}{1-y}\left(z-\varepsilon_{s, p}^{\mathrm{C}}\right) \text {. }
$$

Substituting Eq. (5) into (1) we can write the averaged Green function $\boldsymbol{G}(z)$ in the form

$$
G(z)=T \hat{G} T=T\left(z-\widehat{H}_{0}\right)^{-1} T,
$$

where the transformation matrix $T$ is diagonal with matrix elements 1 for anion sites and $\sqrt{1-y}$ for (all) cation sites. The transformed Hamiltonian $\widehat{\boldsymbol{H}}_{0}$ has the same structure as the Hamiltonian $\boldsymbol{H}_{0}$ for a perfect SnTe crystal with

(i) diagonal matrix elements equal to atomic levels $\varepsilon_{s, p}^{\mathrm{A}}, \varepsilon_{s, p}^{\mathrm{C}}$;

(ii) hopping elements in the anion sublattice unchanged;

(iii) all hoppings in the cation sublattice scaled with a factor $(1-y)$;

(iv) hopping elements between anion and cation states scaled with a factor $\sqrt{1-y}$.

According to (6) the poles of $G(z)$ are just the eigenvalues $E(k)$ of the (periodic) Hamiltonian $\widehat{\boldsymbol{H}}_{0}$ parametrically depending on the vacancy concentration $y$. The Hamiltonian $\widehat{\boldsymbol{H}}_{0}$ represents an alternative, mean-field description of a non-stoichiometric system in which, instead of infinite barriers at the vacant sites, the number of accessible bonding partners is included into the renormalized hopping elements.

Equation (6) also shows that the contribution of cations to the total density of states in $\mathrm{Sn}_{1-y} \square_{y} \mathrm{Te}$ scales as $1-y$. This means that the number of electron states below the band gap, obtained by summing over Te $5 s$ band, Sn $5 s$ band and three Te $5 p$ bands, is $2+2(1-y)+6=10-2 y$ per unit cell. At the same time, the number of electrons per unit cell is $6+4(1-y)=10-4 y$. A cation vacancy introduces just two holes so that, with the lattice constant of SnTe equal to $0.628 \mathrm{~nm}$, the hole density is $p=323 \times 10^{20} y$. We also see that the "acceptor states" are distributed over the whole valence band. 


\section{Results and discussion}

The numerical calculations were done for parametrization from Ref. [4] which gives a reasonable fit to the band structure of SnTe. Using first the mean-field model (6), we find the following dependence of the valence band maxima (in $\mathrm{eV}$, relative to the vacuum level) on the concentration of vacancies and on the density of holes, respectively:

$$
\begin{aligned}
& E(L)=-7.543-0.898 y=-7.543-2.78 \times 10^{-21} p, \\
& E(\Sigma)=-7.781-0.744 y=-7.781-2.30 \times 10^{-21} p .
\end{aligned}
$$

These results were checked by a full CPA calculations for $y=0.1$ with $\varepsilon_{s, p}^{\square}=\infty$ and with more realistic $\varepsilon_{s, p}^{\square}$ close to the vacuum level. The maxima of the spectral density, defining an effective band structure, coincide with $(7,8)$ in both cases.

One can see that, although the relative position of $E(L)$ and $E(\Sigma)$ does not change very much, the absolute downward shift of the valence band energies is comparable with the characteristic parameters from Ref. [1]. This means that not only the Fermi energy moves away from the Gd $d$-level faster than it can be deduced from the increasing hole density, but also that at some vacancy concentration $\varepsilon_{d}$ leaves the heavy-hole band. This seems to be the real explanation of the strong decrease in the AF coupling for highest hole densities. Quantitatively, the change of the hole density $\delta p \approx 2 \times 10^{2} 0 \mathrm{~cm}^{-3}$ (i.e. $\delta y \approx 0.006$ ) is expected to have the same effect as the change of Gd content by one percent. This is in a very good agreement with Fig. 1 of Ref. [1].

In fact, the effect of vacancies on the states close to the valence band top must be similar to the effect due to substitution of $\mathrm{Gd}$. In both cases, cation $s$-states are transferred from the deep valence band into the conduction band. The hybridization of the uppermost valence band with the $s$-states is then partly compensated, which results in the shift of the band to lower energies (cf. [2] for a similar case of $(\mathrm{Cd}, \mathrm{Pb}) \mathrm{S})$.

The CPA calculation also shows a remarkable disorder-induced level broadening $\left(\operatorname{Im} \Sigma_{s, p}^{\mathrm{C}} \approx 1 \mathrm{eV}\right)$ inside the heavy-hole band due to scattering at the vacancies. The disorder may influence the hybridization of the $\mathrm{Gd} d$-state with the band states, and consequently the width of the resulting resonance.

\section{Acknowledgment}

This work was motivated by discussions at the XXV Int. School on Physics of Semiconducting Compounds "Jaszowiec '96". It was supported by the Grant Agency of the Czech Republic under the project number 202/95/0008.

\section{References}

[1] T. Story, M. Górska, A. Łusakowski, M. Arciszewska, W. Dobrowolski, E. Grodzicka, Z. Gołacki, R.R. Gałązka, Phys. Rev. Lett. 77, 3447 (1996).

[2] J. Mašek, J. Phys. C, Solid State Phys. 21, 2821 (1988).

[3] W.A. Harrison, Electronic Structure and the Properties of Solids, Freeman, San Francisco 1980.

[4] J. Robertson, Phys. Rev. B 28, 4671 (1983). 